\title{
A Note on a Recent Attempt to Improve the Pin-Frankl Bound $\|^{*}$
}

\author{
François Gonze $^{1} \quad$ Raphaël M. Jungers $\|^{\dagger} \quad$ Avraham N. Trahtman ${ }^{2}$ \\ 1 ICTEAM Institute, UCLouvain, Louvain La Neuve, Belgium \\ 2 Dep. of Math., Bar-Ilan University, Ramat Gan, Israel
}

received $3^{\text {rd }}$ Dec. 2014, revised $2^{\text {nd }}$ Apr. 2015, $8^{\text {th }}$ Apr. 2015, accepted $8^{\text {th }}$ Apr. 2015.

We provide a counterexample to a lemma used in a recent tentative improvement of the Pin-Frankl bound for synchronizing automata. This example naturally leads us to formulate an open question, whose answer could fix the line of the proof, and improve the bound.

Keywords: Automata, Synchronization, Černý's conjecture.

This short note studies a problem related with synchronizing automata and Černý's conjecture, formulated in [2]. A good survey on the topic is given in [10]. See [1], [4], [5] for recent work on the subject.

A (deterministic, finite state, complete) automaton (DFA) is a triplet $(Q, \Sigma, \delta)$ with $Q$ the set of states, $\Sigma$ the alphabet of letters and $\delta$ the transition function $\delta: Q \times \Sigma \rightarrow Q$ defining the effect of the letters on the states. For $q_{i}, q_{j} \in Q$ and $l \in \Sigma$, we write $q_{i} l=q_{j}$ if $\delta\left(q_{i}, l\right)=q_{j}$. We call a word $w$ of length $m$ a sequence of $m$ letters $l_{1} \ldots l_{m}, l_{i} \in \Sigma, 1 \leq i \leq m$. We write $\Sigma^{m}$ the set of words of length $m$. For $q_{i}, q_{j} \in Q$ and $w=l_{1} \ldots l_{m} \in \Sigma^{m}$, we write $q_{i} w=q_{j}$ if $\delta\left(\ldots \delta\left(\delta\left(q_{i}, l_{1}\right), l_{2}\right) \ldots, l_{m}\right)=q_{j}$. For an automaton with $n$ states and a word $w$, we note $Q w=\left\{q_{j} \mid q_{i} w=q_{j}, 1 \leq i \leq n\right\}$ the set of states that are in the image of $w$. We can represent an automaton as a directed graph. Each state is represented as a vertex, and the effect of each letter on each state is represented as a directed edge. We call a DFA strongly connected if its graph representation is a strongly connected graph.

A word $w$ is called a synchronizing word if, for any states $q_{i}, q_{j} \in Q, q_{i} w=q_{j} w$. A DFA is called a synchronizing automaton if it has a synchronizing word.

Černý's conjecture [2] states that any synchronizing automaton with $n$ states has a synchronizing word of length at most $(n-1)^{2}$.

So far the best proven bound is $\left(n^{3}-n\right) / 6$, obtained more than 30 years ago in [3] and [7], and rediscovered independently in [6]. Recently, a tentative improvement to $n\left(7 n^{2}+6 n-16\right) / 48$ has been proposed in [8]. However, as mentioned later by the author on ArXiv [9], there is a flaw in the proof. Nevertheless, since the publication of [8], many new papers are citing this result, and no publication clearly

*This work was also supported by the communauté francaise de Belgique - Actions de Recherche Concertées and by the Belgian Program on Interuniversity Attraction Poles initiated by the Belgian Federal Science Policy Office. Emails: francois.gonze@uclouvain.be, raphael.jungers@uclouvain.be, trakht@macs.biu.ac.il

${ }^{\dagger}$ R. M. Jungers is a F.R.S.-FNRS Research Associate.

1365-8050 @ 2015 Discrete Mathematics and Theoretical Computer Science (DMTCS), Nancy, France 
confirms that the proof is not valid. In this note, we make this point clear by providing a counterexample to Lemma 3 in [8]. The lemma is the following:

Lemma 1 (Lemma 3 in [8] ) Let $Q$ be the set of states of a synchronizing strongly connected n-state DFA. Then for any state $q$ there exists a word $w$ of length not greater than $n$ such that $q \notin Q w$. For any $k<n$ there are at least $k$ states $q_{1}, \ldots, q_{k}$ and words $w_{1}, \ldots, w_{k}$ of length not greater than $k$ such that $q_{i} \notin Q w_{i}$, $1 \leq i \leq k$.

We refute the lemma by exhibiting an automaton such that, for one state $q_{0}$, there is no word $w$ of length smaller than or equal to the number of states with the property that $q_{0} \notin Q w$.

Counterexample The automaton represented in Fig.11 is a synchronizing automaton, as the word $a b b a b a b b a$ is a synchronizing word. However, the shortest word $w$ such that $q_{0} \notin Q w$ is $w=a b b a b a$.

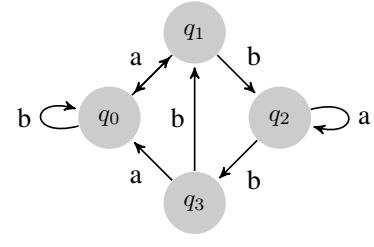

Since the automaton has only 4 states and $w$ is 6 letters long, this contradicts Lemma 1

Lemma 1 was a key step in the improvement on the maximal length of a shortest synchronizing word. We observe that a weaker version of Lemma 1 could still improve the Pin-Frankl bound. In fact, any value proportional to the number of states of the automaton would lead to an improvement of the bound. This motivates us to raise the following open question.

Open question Let $Q$ be the set of states of a synchronizing strongly connected n-state DFA.

Is there a constant $c$ such that, for any state $q \in Q$, there exists a word $w$ of length not greater than $\mathrm{cn}$ such that $q \notin Q w$ ?

\section{References}

[1] V. Blondel, R. M. Jungers, and A. Olshevsky. On primitivity of sets of matrices. ArXiv preprint. http://arxiv.org/abs/1306.0729, 2014.

[2] J. Černý. Poznámka k homogénnym eksperimentom s konečnými automatami. Matematicko-fysikalny Casopis SAV, 14:208-216, 1964.

[3] P. Frankl. An extremal problem for two families of sets. European Journal of Combinatorics, 3:125-127, 1982.

[4] F. Gonze and R. M. Jungers. On the synchronizing probability function and the triple rendezvous time for synchronzing automata. ArXiv preprint. http://arxiv.org/abs/1410.4034, 2014.

[5] R. M. Jungers. The synchronizing probability function of an automaton. SIAM Journal on Discrete Mathematics, 26(1):177-192, 2012.

[6] A. A. Klyachko, I. K. Rystsov, and Spivak M. A. An extremal combinatorial problem associated with the bound on the length of a synchronizing word in an automaton. Kibernetika, 2:16-20, 1987. in Russian; Engl. translation: Cybernetics 23 (1987) 165-171.

[7] J.-E. Pin. On two combinatorial problems arising from automata theory. Annals of Discrete Mathematics, 17:535-548, 1983.

[8] A. N. Trahtman. Modifying the upper bound on the length of minimal synchronizing word. In FCT 2011, volume 6914 of Lecture Notes in Computer Science, pages 173-180. Springer-Verlag, 2011.

[9] A. N. Trahtman. Modifying the upper bound on the length of minimal synchronizing word. ArXiv preprint. http://arxiv.org/abs/1104.2409, 2011.

[10] M. V. Volkov. Synchronizing automata and the Černý conjecture. In LATA 2008, volume 5196 of Lecture Notes in Computer Science, pages 11-27. Springer-Verlag, 2008. 